\title{
Efeito do tipo de substrato para pupação na dispersão larval pós- alimentar de Chrysomya albiceps (Diptera, Calliphoridae)
}

\author{
Leonardo Gomes $^{1}$, Guilherme Gomes ${ }^{1}$, Helena G. Oliveira ${ }^{1}$, Claudio J. Von Zuben ${ }^{1}$, \\ Iracema M. da Silva ${ }^{1} \&$ Marcos R. Sanches ${ }^{2}$
}

1. Departamento de Zoologia, Universidade Estadual Paulista, Av. 24A, 1515, 13506-900 Rio Claro, SP, Brasil. (leugomes@yahoo.com.br) 2. Rua do Rocio 220, $1^{\circ}$ andar, Vila Olímpia, 04552-903 São Paulo, Brasil. (marcos.sanches@ipsos.com.br)

\begin{abstract}
Effect of the substrate for pupation in the postfeeding larval dispersal of Chrysomya albiceps (Diptera: Calliphoridae). Blowflies utilize discrete and ephemeral sites for breeding and larval nutrition. After the exhaustion of food, the larvae begin dispersing to search for pupation sites or for additional food sources, a process referred to as postfeeding larval dispersal. However the nature of the substrate can affect this process. Some of the most important aspects of this behavior were here investigated in Chrysomya albiceps (Wiedemann, 1819), utilizing a circular arena covered with vermiculite allowing the post-feeding larval dispersal from the center and to comparing with others studies that used sawdust. To determinate the location of each pupa, the arena was divided into 72 equal sectors from the center. For each pupa, weight, dispersal distance from the center of arena, and depth were determined. Statistical tests were performed to verify the relationship among weight, depth and distance of burial for pupation. The larvae that dispersed farthest were those with lower weights, as in other studies that employed other substrates. The majority of individuals reached the burial depth for pupation between 2 and $8 \mathrm{~cm}$ (mean $4.96 \pm 2.97 \mathrm{~cm}$ ). The study of this dispersal process can be helpful in the estimation of postmortem interval (PMI) for human corpses in medico-criminal investigations.
\end{abstract}

KEYWORDS. Blowflies, Calliphoridae, Chrysomya albiceps, substrate for pupation, larval dispersal.

RESUMO. As moscas-varejeiras utilizam substratos discretos e efêmeros para posturas dos ovos e para alimentação das larvas. Após a exaustão de recursos, as larvas começam a procurar por um sítio de pupação no habitat ou por mais fonte de alimento adicional (dispersão larval pós-alimentar). No entanto, o tipo de substrato de dispersão pode afetar este processo; assim, procurou-se avaliar o comportamento de dispersão de Chrysomya albiceps (Wiedemann 1819) por meio da localização das pupas em arenas circulares preenchidas com vermiculita, comparando-se com outros estudos feitos em serragem. As arenas foram dividas em 72 setores iguais a partir do centro de dispersão para facilitar a localização das pupas no substrato. A massa, a distância de dispersão do centro de arena, e a profundidade de cada pupa foram determinadas e verificou-se a relação entre peso, profundidade e distância do centro de dispersão. Pôde-se constatar que as larvas com menor massa foram as que percorreram maior distância do centro de dispersão, como também foi observado em estudos anteriores que empregaram outros substratos. O sítio de pupação da maioria dos indivíduos foi entre 2 e 8 cm de profundidade (média 4,96 2,97 cm). Tal estudo do processo de dispersão pode ser útil na estimativa do intervalo pós-morte (IPM) para corpos humanos em investigações médico-criminais.

PALAVRAS-CHAVE. Calliphoridae, Chrysomya albiceps, dispersão larval, moscas-varejeiras, substrato para pupação.

As moscas do gênero Chrysomya têm grande importância médico-veterinária por serem veiculadoras de enteropatógenos tais como vírus, bactérias e helmintos (FURLANETTO et al., 1984), podendo causar também miíases nos animais e no homem (ZuMPT, 1965; GuIMARÃEs et al., 1983). São também de fundamental importância em entomologia forense por serem indicadoras de tempo de decomposição de cadáveres humanos (Wells \& GREenBERG, 1992; Von Zuben et al., 1996; GoMEs \& Von ZuBEN, 2004).

Chrysomya albiceps (Wiedemann, 1819), originária do continente africano, foi introduzida acidentalmente no continente americano, provavelmente através de navios (GUIMARÃes et al., 1978). Em 1975, esta espécie foi descoberta no sudeste do Brasil juntamente com $C$. albiceps (Wiedemann) e C. chloropyga (Wiedemann, 1819) (IMBiRIBa et al., 1977; Guimarães et al., 1978).

Essas espécies de moscas-varejeiras utilizam-se de substratos discretos e efêmeros para posturas dos ovos e para alimentação das larvas (HANSKI, 1987; PESCHKE et al., 1987). O estágio larval é o principal período em que ocorre limitação de recursos alimentares e a competição por esses recursos é, geralmente, do tipo exploratório
(ReIs et al., 1994), onde cada larva procura ingerir o máximo de alimento possível antes da completa exaustão dos recursos (UlLYETT, 1950). Após essa competição, as larvas começam a procurar um sítio para pupação ou por mais fontes de alimento (no caso de larvas que não obtiveram o peso mínimo para a pupação) (Gomes et al., 2002); tal processo é denominado dispersão larval pósalimentar (GREENBERG, 1990).

Alguns estudos sobre dispersão larval pósalimentar em C. albiceps já foram feitos com a intenção de se observar padrões de distribuição, bem como a análise das variáveis em estudo durante o comportamento de busca por substrato para pupação ou por novas fontes de alimento (Gomes et al., 2002). Nesses estudos (Gomes et al. 2002, 2003; Gomes \& Von Zuben, 2003) discutiu-se a possibilidade da influência do substrato no processo de dispersão e enterramento para pupação à semelhança do que ocorre na natureza.

Nesse contexto, objetivou-se estudar sob condições experimentais, em simulação de ambiente natural, a dispersão larval pós-alimentar em C. albiceps em uma arena com vermiculita. Com isso, tentou-se estabelecer uma comparação com outros estudos (GOMES 
\& Von Zuben, 2005), onde foi usada serragem, além de estabelecer uma relação entre as variáveis peso, distância e profundidade de enterramento.

\section{MATERIAL E MÉTODOS}

Exemplares adultos de $C$. albiceps foram coletados no Instituto de Biociências da UNESP, Rio Claro, SP, utilizando como isca carcaça de peixe. Os exemplares coletados foram identificados e mantidos em gaiolas teladas, em sala com temperatura de $25 \pm 1^{\circ} \mathrm{C}, 60 \pm 10 \%$ de umidade relativa e fotofase de 12 horas, sendo ministrados água, açúcar e fígado ad libitum.

Uma arena circular (50 $\mathrm{cm}$ de diâmetro) foi montada sobre o piso de uma sala do laboratório iluminada de forma homogênea (a fim de evitar tendências na dispersão das larvas), sendo forrada com vermiculita, tendo suas margens delimitadas por papelão e dividida em 72 setores de 5 graus cada. O desenvolvimento larval deu-se em carne moída bovina até o $3^{\circ}$ instar, sendo este substrato então colocado no centro da arena circular forrada com vermiculita, para que as 274 larvas (média dos três experimentos em que foram usadas 274,270 e 278 larvas para cada um) abandonassem a mesma e irradiassem para empupar.

Para o estudo da dispersão larval pós-alimentar foram realizados três experimentos com as arenas forradas com uma camada de $20 \mathrm{~cm}$ de vermiculita, que serviu para investigar qual a profundidade máxima em que as larvas se enterravam para empupar e comparar o efeito do tipo de substrato com estudos anteriores.

As pupas foram localizadas, retiradas da vermiculita, determinando-se sua localização por setor e medindo-se para cada uma, a distância e a profundidade do local de pupação com o auxílio de régua ou trena. A coleta das pupas começou da periferia da arena para o centro; cada uma delas foi pesada em balança analítica e individualizada em frascos de plástico, antes que ocorresse a emergência do adulto. Os indivíduos adultos foram sexados e o material-testemunho está depositado na coleção entomológica do Departamento de Zoologia da UNESP, Rio Claro.

Foram feitos testes estatísticos de regressão, correlação, qui-quadrado $\left(\chi^{2}\right)$ e igualdade de médias (ANOVA) a $95 \%$ de confiança, bem como análises direcionais para todas as variáveis estudadas (ZAR, 1999).

\section{RESULTADOS}

O quarto quadrante concentrou uma porcentagem bem menor das larvas, ocorrendo após mais ou menos o raio de 220 graus, no terceiro quadrante (x e y negativos) (Fig. 1). A disposição das larvas por quadrante foi o seguinte: primeiro (29\%), segundo (31\%), terceiro (23\%), quarto $(17 \%)$ (Fig. 1). A distribuição da direção escolhida pelas larvas na arena é aleatória (as larvas se enterraram em todas as direções). Em algumas direções houve uma concentração de larvas, mas poucas após os 90 e 180 graus (Fig. 1). A direção média foi de 96 graus, o que pode ser explicado pela existência de um grupo de larvas pouco após os 180 graus, contrabalanceado por outra concentração no lado oposto. Dadas as concentrações de larvas em várias direções, nenhuma destas predominou sendo o vetor médio pequeno $(0,196$ de uma variação entre zero e um). Em acréscimo, o teste de Rao acusou não-uniformidade da direção escolhida pelas larvas. Isto provavelmente ocorreu porque se observaram grupos de larvas, bem como alguns setores vazios.

A distribuição da distância percorrida pelas larvas a partir do centro da arena foi de no máximo $25 \mathrm{~cm}$ (Fig. 2). Verificou-se que a maior parte das larvas ocorreu a uma distância inferior a $15 \mathrm{~cm}$, sendo que menos de $10 \%$ delas foram além dessa distância; a média foi de 7,6 $4,95 \mathrm{~cm}$.

A distribuição do peso das larvas (Fig. 2) demonstrou que a maioria das pupas coletadas pesou entre 47 e $63 \mathrm{mg}$, sendo que menos de $10 \%$ das larvas pesaram menos de $47 \mathrm{mg}$ e o peso médio foi de $54,6 \pm 5,0 \mathrm{mg}$.

Apenas duas larvas se enterraram a profundidades maiores que $15 \mathrm{~cm}$ (Fig. 2), sendo a profundidade média de 4,96 $\pm 2,97 \mathrm{~cm}$, com as larvas concentrando-se entre 2 $\mathrm{e} 12 \mathrm{~cm}$

A maior parte das larvas com peso menor (67\%) percorreu maior distância; para as larvas com peso intermediário, $68 \%$ deslocaram-se por uma distância média, e finalmente para a maioria das larvas mais pesadas (67\%) a distância percorrida foi menor (Fig. 1).

Peso e distância estão significativamente relacionados $\left(\chi^{2}=146,4 ; \mathrm{gl}=4\right)(\mathrm{Tab} . \mathrm{I})$ sendo que o coeficiente de correlação (r) entre distância e peso foi de -0.51 (Correlação de Pearson, $\mathrm{p}=0,0034$ ). Além disso, quanto maior a distância e quanto menor o peso, maior a profundidade média de enterramento das larvas $(r=0,41$, $p<0,001 ; r=-0,31, p<0,001$ respectivamente)

$\mathrm{Na}$ tentativa de se prever a profundidade de enterramento de uma pupa, dado o seu peso, seu sexo e a distância percorrida, foi feita uma análise de regressão. A equação final foi: Profundidade $=10,023+$ 0,119*Distância - $1,097 *$ Sexo M - 0,101*Peso.

Embora o sexo das larvas não tenha influência significativa na profundidade de forma isolada (Tab. II) conjuntamente com as outras variáveis (em um modelo

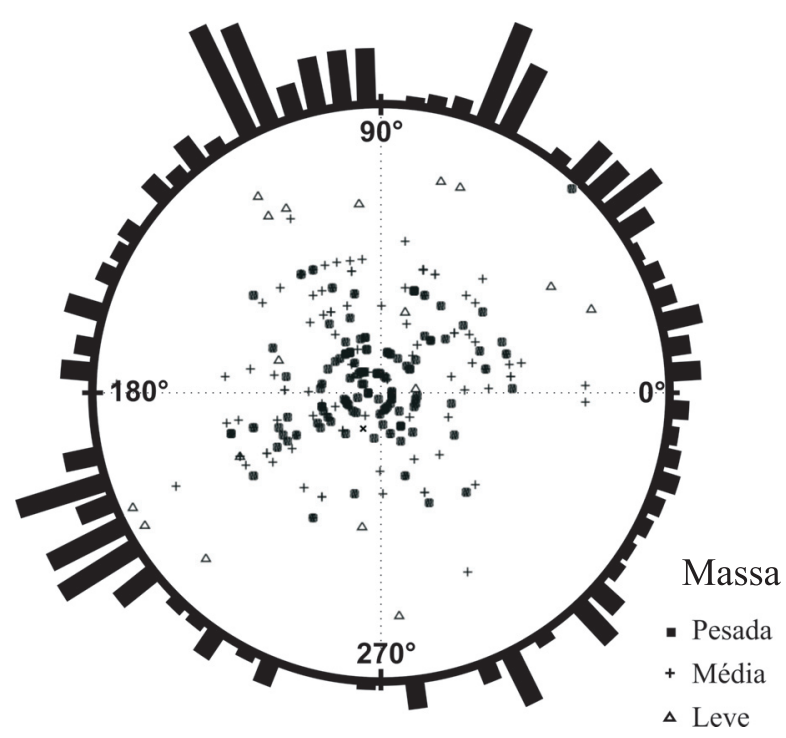

Fig. 1. Distribuição das larvas de Chrysomya albiceps (Wiedemann 1819) na arena de acordo com as faixas de peso (mg) e a direção escolhida (histogramas ao redor da arena). 
de regressão) o sexo é importante. Quando fixadas a distância e o peso, a profundidade média para o sexo masculino é $1 \mathrm{~cm}$ menor do que para o sexo feminino. Este fator vale apenas para uma mesma distância e peso; a profundidade média para ambos os sexos não apresentou diferença significativa, se forem considerados os dados de uma forma geral (Tab. II). $\mathrm{O} \mathrm{r}^{2}$ de 0,364 do modelo é baixo, o que indica que o modelo, se usado para fins de predição, não é muito eficiente com relação ao sexo, mas o é com relação à distância e peso.

Embora a diferença nas médias das variáveis analisadas das larvas entre os experimentos não tenham sido significativas, deve se levar em conta a amostra razoavelmente numerosa, de tal sorte que o teste estatístico é eficiente para detectar até pequenas diferenças de média (ANOVA, p<2,59 a 5\% de significância).

Tabela I. Distribuição percentual entre as faixas de peso (mg) e de distância (cm) das larvas de Chrysomya albiceps (Wiedemann, 1819) e seu coeficiente de correlação*. * Coeficiente de correlação de Pearson $=-0.51(\mathrm{p}=0,00034)$.

\begin{tabular}{|c|c|c|c|c|c|}
\hline & & Distância menor & Distância média & Distância maior & Total \\
\hline \multirow[t]{3}{*}{ Larvas leves } & Freqüência & 2,0 & 4,0 & 12,0 & 18,0 \\
\hline & $\%$ dentro do peso & $11,1 \%$ & $22,2 \%$ & $66,7 \%$ & $100,0 \%$ \\
\hline & \% dentro da distância & $1,6 \%$ & $3,1 \%$ & $63,2 \%$ & $6,6 \%$ \\
\hline \multirow[t]{3}{*}{ Larvas intermediárias } & Freqüência & 35,0 & 85,0 & 6,0 & 126,0 \\
\hline & $\%$ dentro do peso & $27,8 \%$ & $67,5 \%$ & $4,8 \%$ & $100,0 \%$ \\
\hline & \% dentro da distância & $28,2 \%$ & $64,9 \%$ & $31,6 \%$ & $46,0 \%$ \\
\hline \multirow[t]{3}{*}{ Larvas pesadas } & Freqüência & 87,0 & 42,0 & 1,0 & 130,0 \\
\hline & $\%$ dentro do peso & $66,9 \%$ & $32,3 \%$ & $0,8 \%$ & $100,0 \%$ \\
\hline & \% dentro da distância & $70,2 \%$ & $32,1 \%$ & $5,3 \%$ & $47,4 \%$ \\
\hline \multirow[t]{3}{*}{ Total } & Freqüência & 124,0 & 131,0 & 19,0 & 274,0 \\
\hline & $\%$ dentro do peso & $45,3 \%$ & $47,8 \%$ & $6,9 \%$ & $100,0 \%$ \\
\hline & \% dentro da distância & $100,0 \%$ & $100,0 \%$ & $100,0 \%$ & $100,0 \%$ \\
\hline
\end{tabular}

Tabela II. Médias e desvios padrões das pupas coletadas de Chrysomya albiceps (Wiedemann, 1819) na arena, considerando-se os três experimentos.

\begin{tabular}{|c|c|c|c|c|c|c|}
\hline & SEXO & Média & Desvio Padrão & Mínimo & Máximo & $\mathrm{N}^{\mathrm{o}}$ de Pupas \\
\hline \multirow[t]{4}{*}{ PESO (mg) } & Não Identificado & 56,17 & 4,38 & 48,2 & 61,2 & 14 \\
\hline & Fêmea & 54,97 & 5,04 & 41,4 & 62,0 & 142 \\
\hline & Macho & 54,02 & 5,01 & 40,4 & 61,4 & 118 \\
\hline & Total & 54,63 & 5,01 & 40,4 & 62,0 & 274 \\
\hline \multirow[t]{4}{*}{ DISTÂNCIA (cm) } & Não Identificado & 6,53 & 3,52 & 2,0 & 12,0 & 14 \\
\hline & Fêmea & 7,66 & 5,23 & 0,0 & 25,0 & 142 \\
\hline & Macho & 7,64 & 4,76 & 0,0 & 21,0 & 118 \\
\hline & Total & 7,59 & 4,95 & 0,0 & 25,0 & 274 \\
\hline \multirow[t]{4}{*}{ PROFUNDIDADE $(\mathrm{cm})$} & Não Identificado & 5,27 & 3,13 & 2,0 & 12,0 & 14 \\
\hline & Fêmea & 5,39 & 3,18 & 0,0 & 20,0 & 142 \\
\hline & Macho & 4,39 & 2,60 & 0,0 & 15,0 & 118 \\
\hline & Total & 4,96 & 2,97 & 0,0 & 20,0 & 274 \\
\hline
\end{tabular}
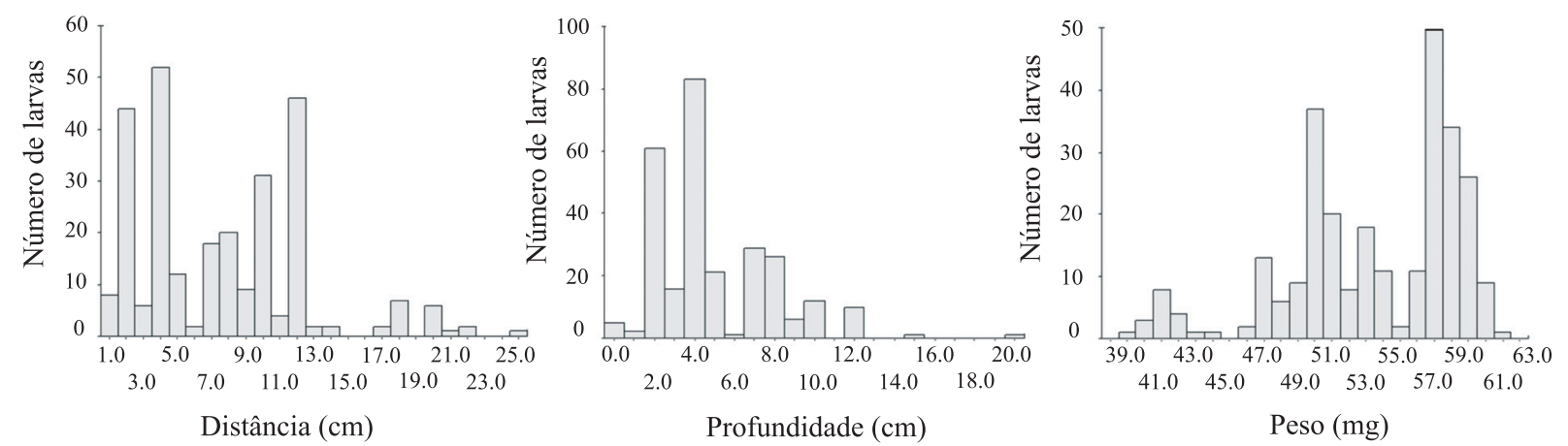

Fig. 2. Distribuição das pupas de Chrysomya albiceps (Wiedemann, 1819) na arena de acordo com a distância, profundidade de enterramento $(\mathrm{cm})$ e peso $(\mathrm{mg})$ das larvas. 


\section{DISCUSSÃO}

Este estudo descreveu aspectos gerais do processo de dispersão de larvas em uma arena circular e confirmou resultados anteriores utilizando arenas com mesmo diâmetro e mesma profundidade (GOMEs \& Von ZuBEN, 2005), porém com serragem, em que as larvas de $C$. albiceps com peso menor são as que têm tendência de se deslocar mais. Tal fato explica-se por estarem as larvas à procura de nova fonte alimentar e não buscando distâncias maiores para se enterrarem e empupar (GOMES \& Von ZuBEN, 2003; Gomes et al., 2002, 2003). A correlação negativa entre a distância e o peso das pupas está de acordo com outro experimento realizado em serragem (Gomes \& Von Zuben, 2003) onde também se observou que o peso diminui com a distância.

A maior concentração de pupas foi observada nas profundidades entre 4 e $8 \mathrm{~cm}$ e apenas duas larvas atingiram a profundidade máxima a partir da superfície $(20 \mathrm{~cm})$. Aparentemente, o que mais limitou a movimentação das larvas foi a profundidade para enterramento a partir do centro, bem como a granulometria maior da vermiculita quando comparada com a serragem.

As larvas atingiram menores profundidades de

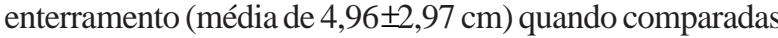
ao estudo de Gomes \& Von ZuBEN (2005) (10,93 cm). Porém, de uma maneira geral o tipo de substrato não interfere no comportamento de dispersão das larvas de C. albiceps, uma vez que elas se distribuíram aleatoriamente e nas mesmas distâncias médias $(7,59 \mathrm{~cm})$. Tal média foi bem próxima de estudos anteriores realizados por Gomes \& Von ZuBEN (2005) (8,21 cm), apenas confirmando um comportamento esperado que, na presença de obstáculos para empupar, as larvas se enterram menos profundamente.

O presente estudo reflete melhor as condições de ambiente natural que as larvas vão enfrentar ao abandonar o substrato alimentar em busca de um sítio para pupação por empregar uma arena circular (ULLYETT, 1950). Esta última permite uma dispersão radial das larvas a partir do substrato alimentar localizado no centro (e não simplesmente uma dispersão numa única direção e em apenas dois sentidos, como no caso de uma calha).

O comportamento de dispersão larval pós-alimentar e o consequiente padrão de distribuição espacial das pupas nos sítios de pupação podem ter implicações na susceptibilidade das mesmas ao ataque de predadores e parasitóides em populações de ambientes naturais (LeGNer, 1977; PeschKe et al., 1987).

Estudos de dispersão podem ter importantes implicações para investigações médico-criminais, porque a presença de larvas e pupas em cadáveres humanos e circunvizinhanças podem auxiliar na estimativa do intervalo de tempo entre a morte e a descoberta do cadáver, referido como intervalo pós-morte (IPM). Essa estimativa constitui um dos aspectos fundamentais nos estudos de medicina legal (Sмiтн, 1986), sendo que a mesma pode ser seriamente prejudicada levando a uma subestimativa do IPM, caso não sejam consideradas as larvas dispersantes que se enterraram mais afastadas do cadáver (GOMES \& VoN Zuben, 2004, 2005; GoMes et al., 2006).
Agradecimentos. Os autores agradecem à FAPESP pelo auxílio financeiro para a realização desses estudos (Processo 03/ 00540-3), ao CNPq pela bolsa de pesquisa do quarto autor, aos revisores anônimos pelas sugestões e à editora assistente pela correção.

\section{REFERÊNCIAS BIBLIOGRÁFICAS}

Furlanetto, S. M. P.; Campos, M. L. C.; Hársi, C. M.; Buralli, G. M. \& Ishinata, G. K. 1984. Microrganismos enteropatogênicos em moscas africanas pertencentes ao gênero Chrysomya (Diptera: Calliphoridae) no Brasil. Revista de Microbiologia 15(3): 170-174.

Gomes, L. \& Von Zuben, C. J. 2003. Distribuição larval radial pósalimentar em Chrysomya albiceps (Wied.) (Diptera: Calliphoridae): profundidade, distância e peso de enterramento para pupação. Entomologia y Vectores 10:211-222.

2004. Insetos ajudando a desvendar crimes: Entomologia forense. Ciência Hoje 208:28-31.

2005. Postfeeding radial dispersal in larvae of Chrysomya albiceps (Diptera: Calliphoridae): implications for forensic entomology. Forensic Science International 155:61-64.

Gomes, L.; Von Zuben, C. J. \& Govone, J. S. 2002. Comportamento da dispersão larval radial pós-alimentar em moscas-varejeiras do gênero Chrysomya (Diptera: Calliphoridae): busca por novas fontes de alimento. Entomologia y Vectores 9(1):115-132.

Gomes, L; Von Zuben, C. J. \& SAnches, M. R. 2003. Estudo da dispersão larval radial pós-alimentar em Chrysomya megacephala (Fabricius) (Diptera: Calliphoridae). Revista Brasileira de Entomologia 47(2):229-234.

Gomes L.; Godoy, W. A. \& Von Zuben, C. J. 2006. Postfeeding larval dispersal: implications to forensic entomology. Naturwissenschaften 93:207-215.

GreEnBerG, B. 1990. Behavior of postfeeding larvae of some Calliphoridae and a muscid (Diptera). Annals of Entomological Society of America 83:1210-1214

Guimarães, J. H.; Prado, A. P. \& Linhares, A. X. 1978. Three newly introduced blowfly species in Southern Brazil (Diptera: Calliphoridae). Revista Brasileira de Entomologia 22 (1):53-60.

Guimarães, J. H.; Papavero, N. \& Prado, A. P. 1983. As miíases na região neotropical (identificação, biologia, bibliografia). Revista Brasileira de Zoologia 1(4):239-416.

HANSKI, I. 1987. Carrion fly community dynamics: patchiness, seasonality and coexistence. Ecological Entomology 12:257-266.

Imbiriba, A. S.; Izutani, D. T.; Milhoreto, I. T. \& LuZ, E. 1977. Introdução da Chrysomya chloropyga (Wiedemann, 1818) na região Neotropical (Diptera: Calliphoridae). Arquivos de Biologia e Tecnologia 20:35-39.

Legner, E. F. 1977. Temperature, humidity and depth of habitat influencing host destruction and fecundity of muscoid fly parasites. Entomophaga 22:199-206.

PeschKe, K.; Krapf, D. \& Fuldner, D. 1987. Ecological separation, functional relationships, and limiting resources in a carrion insect community. Zoologische Jahrbücher 114:241-265.

Reis, S. F.; Stangenhaus, G.; Godoy, W. A. C.; Von Zuben, C. J. \& Ribeiro, O. B. 1994. Variação em caracteres bionômicos em função de densidade larval em Chrysomya megacepala e Chrysomya putoria (Diptera: Calliphoridae). Revista Brasileira de Entomologia 38(1):33-34

Sмiтн, K. G. V. 1986. A manual of forensic entomology Ithaca, Cornell University Press.

Ullyett, G. C. 1950. Competition for food and allied phenomena in sheep-blowfly populations. Philosophical Transactions of Royal Society of London 234:77-174.

Von Zuben, C. J.; Bassanezi, R. C. ; dos Reis, S. F.; Godoy, W. A. C. \& Von Zuben, F. J. 1996. Theoretical approaches to forensic entomology: I. Mathematical model of postfeeding larval dispersal. Journal of Applied Entomology 120:379-382.

Wells, J. D. \& Greenberg, B. 1992. Interaction between Chrysomya rufifacies and Cochliomyia macellaria (Diptera: Calliphoridae): the possible consequences of an invasion. Bulletin of Entomological Research 82:133-137.

ZAR, J. H. 1999. Biostatistical analysis. New Jersey. Prentice Hall. 666p.

Zumpt, F. 1965. Myiasis in man and animals in the Old World. London, Butterworths. 267p. 